\title{
Microfluidic Lateral Flow Cytochrome P450 Assay on a Novel Printed Functionalized Calcium Carbonate-Based Platform for
} Rapid Screening of Human Xenobiotic Metabolism

\author{
Jutila, Eveliina
}

2018-08-01

Jutila , E , Koivunen , R , Kiiski , I , Bollström , R , Sikanen , T \& Gane , P 2018 , '

Microfluidic Lateral Flow Cytochrome P450 Assay on a Novel Printed Functionalized

Calcium Carbonate-Based Platform for Rapid Screening of Human Xenobiotic Metabolism ' ,

Advanced Functional Materials , vol. 28 , no. 31 , 1802793 . https://doi.org/10.1002/adfm.201802793

http://hdl.handle.net/10138/303908

https://doi.org/10.1002/adfm.201802793

submittedVersion

Downloaded from Helda, University of Helsinki institutional repository.

This is an electronic reprint of the original article.

This reprint may differ from the original in pagination and typographic detail.

Please cite the original version. 


\section{WILEY-VCH}

DOI: 10.1002/ ((please add manuscript number))

Article type: Full Paper

\section{Microfluidic Lateral Flow Cytochrome P450 Assay on Printed Functionalized Calcium Carbonate-based Platform for Rapid Screening of Human Xenobiotic Metabolism}

Eveliina Jutila*, Risto Koivunen, Iiro Kiiski, Roger Bollström, Tiina Sikanen*, Patrick Gane

E. Jutila, R. Koivunen, Dr. R. Bollström, Prof. P. Gane

Department of Bioproducts and Biosystems

Aalto University School of Chemical Engineering

Aalto, FI-00076, Finland

E-mail: eveliina.jutila@aalto.fi (material development)

Dr. R. Bollström, Dr. P. Gane

Omya International AG

Oftringen, $\mathrm{CH}-4665$, Switzerland

I. Kiiski, Dr. T. Sikanen

Faculty of Pharmacy

Drug Research Program

University of Helsinki

Helsinki, FI-00014, Finland

Email: tiina.sikanen@ helsinki.fi (CYP assay design)

Keywords: porous materials, functional coatings, inkjet printing, drug metabolism, cytochrome $\mathrm{P} 450$

Cytochrome P450 (CYP) is a superfamily of enzymes in charge of elimination of the majority of clinically used drugs and other xenobiotics. This study focuses on the development of a rapid microfluidic lateral flow assay to study human phase I metabolism reactions mediated by CYP2A6 isoenzyme, the major detoxification route for many known carcinogens and drugs, with coumarin 7-hydroxylation, as the prototype model reaction. The lateral flow assay is fabricated utilizing custom-designed porous functional calcium carbonate (FCC) based substrates and inkjet-printed fluid barriers. All materials used are carefully characterized with a view to preserve biocompatibility. The final assay comprises separate zones for reaction, separation and detection, and an absorbent pad to keep the assay wet for extended periods (up to $10 \mathrm{~min}$ ) even when heated to physiological temperature. The overall concept allows one to 


\section{WILEY-VCH}

carry out CYP assays at lower cost than conventional well-plate assays, while providing increased selectivity at equally high speed, owing to the possibility for simultaneous chromatographic separation of the reaction products from the reactants on the FCC coating. The developed concept thus provides a viable approach for rapid prediction of the interaction risks related to metabolic clearance of drugs and other xenobiotics.

\section{Introduction}

Modern drug discovery provides an enormous number of new drug candidates, the properties of which need to be carefully characterized as early as possible during the preclinical phase in order to identify the most potential lead compounds for further optimization. The drug discovery cycle from a lead compound to the commercial product is a capital-intensive process, which would greatly benefit from more economic and efficient in vitro tools that are capable of speeding up the preclinical screening. All new chemical entities (NCE) need to be screened for their pharmacokinetic properties, including absorption, distribution, metabolism, excretion and toxicology (ADMET), which forms a bottleneck in the drug discovery cycle. Although many of these steps can be predicted by computer-aided design, the selectivity of enzymatic biotransformation reactions cannot be reproduced reliably by any known non-enzymatic technique $^{[1]}$ and, thus, the metabolic profile needs to be confirmed experimentally. Particularly important is the prediction of the metabolic interactions mediated by the cytochrome P450 (CYP) system, which is a superfamily of enzymes accounting for about $75 \%$ of the human phase I metabolism of all clinically used drugs along with endogenous steroid synthesis and detoxification of numerous other xenobiotics. ${ }^{[2]}$ In addition to drug-drug interactions, it is important to examine the risk of metabolic interactions between clinically used drugs and the environmental chemicals for which humans are unintentionally exposed to on a daily basis. 


\section{WILEY-VCH}

Many of such unintentionally uptaken chemicals are small molecules that occupy the CYP system as their main detoxification route (first phase metabolism) same as drugs. ${ }^{[3]}$

Microfluidic paper-based lateral flow assays represent one of the most promising and fastgrowing new fields in bioanalytical chemistry, point-of-care (POC) medical diagnosis, environmental analysis and food quality testing. ${ }^{[4]}$ Paper-based assays offer small, simple-touse, low-cost, portable and disposable platforms exploiting capillary-driven liquid transport. ${ }^{[5]}$ Paper fluidic assays can be patterned using a range of techniques, including photolithography, ${ }^{[5 a, 6]}$ plasma ${ }^{[7]}$ and laser treatment ${ }^{[8]}$ or by using printing methods such as wax printing, ${ }^{[9]}$ screen printing, ${ }^{[6 c, 10]}$ flexography ${ }^{[11]}$ or inkjet. ${ }^{[12]}$ Inkjet printing in particular enables a simple and low-cost mass production of microfluidic devices. ${ }^{[13]}$

Filter and chromatography papers are the most widely used substrates in microfluidic paperbased assays, ${ }^{[14]}$ though such papers have the disadvantages of fibrous and heterogeneous consistency and the coarse scale of their pore structure. In addition to filter and chromatography papers, some researchers have used copy paper, ${ }^{[11 b, 15]}$ but such paper has low wicking capabilities making it, as such, unsuitable for flow assays. ${ }^{[15]}$ Furthermore, commercial papers may contain biocides that inhibit the enzyme activities or other additives that cause high optical background (especially when exposed in the UV range) and, thus, render them unsuitable for trace-level detection of pharmaceuticals and other small molecules. ${ }^{[4 a, 16]}$ Until now, most paperbased biosensors have been developed with a view to colorimetric detection of, for instance, glucose and other abundant biomolecules ${ }^{[5,9 a, 11 a, b, 12 a, b]}$, or of tailor-made antigen-antibody binding. ${ }^{[17]}$ Paper-based assays capable of direct optical detection of the low concentrations of pharmaceuticals and other alike small molecules could provide for beneficial development opportunities. 


\section{WILEY-VCH}

Custom-designed functional materials have recently been developed for use in coatings with a view to microfluidic assays. ${ }^{[11 \mathrm{~b}, 18]}$ The benefit of highly porous coating is its homogeneous surface with a finer and tailorable pore network structure, which allows not only the use of lower sample volumes but also the fabrication of assays with higher feature resolution and more uniform flow characteristics. In this study, a coated lateral flow CYP2A6 assay was fabricated utilizing specially designed porous functionalized calcium carbonate (FCC) as the hydrophilic wicking material, with microfluidic path control defined by inkjet-printed hydrophobic barriers. The coating formulation was optimized to achieve enhanced porosity and elongated wetting capabilities, the components having been carefully examined for their biocompatibility regarding the desired end use as a disposable CYP lateral flow assay. The printing conditions were also optimized to achieve either fully fluid impermeable, or controlled semi-impermeable, barriers to prevent or allow selectively the passage of both eluent toward the reaction zone and the reaction product-containing solution onward to the separation channel and eventually entering the measurement endpoint region. The CYP enzymes were incorporated into the assay in human liver microsomes (HLM) containing all of the main CYP isoforms, and coumarin 7hydroxylation was used as the prototypic model activity for CYP2A6 isoenzyme. CYP2A6 was chosen as the primarily targeted isoenzyme, as it is an important detoxification route for many carcinogens, such as nicotine, ${ }^{[19]}$ and thus a likely target of drug-carcinogen interactions. The performance of the final assay was validated in terms of the dependency between the amounts of the formed metabolite, 7-hydroxycoumarin, and the applied enzyme, which lays the basis for further use of the developed lateral flow assays in determining the enzyme inhibition kinetics (e.g. the $\mathrm{IC}_{50}$ and $K_{\mathrm{i}}$ parameters).

\section{Results and Discussion}

\subsection{Selection of materials}




\section{WILEY-VCH}

Coating fabrication design for the lateral flow CYP assay was initiated by careful characterization of the constituent materials for their compatibility with CYP enzymes using conventional in-solution assays. The effects of FCC and two different binders, i.e. microfibrillated celluloses (MFC A and MFC B), on the chosen CYP2A6 model activity, coumarin 7-hydroxylation, were determined by adding each compound separately to the enzyme incubation solution and comparing the obtained enzyme activity to that of the control incubation. No significant alterations in the enzyme activity were observed upon addition of FCC, MFC A or MFC B, suggesting that the risk of mechanistic inhibition by the chosen coating pigment or the binders is negligible (Figure 1a). The hydrophobizing agents used in printing the fluidic barriers, polystyrene (PS) and alkyl ketene dimer (AKD), were both insoluble in water. Thus, PS or AKD were not considered capable of diffusing into the point of CYP assay, which was to be performed on otherwise non-printed FCC-based coating. In addition, nonspecific adsorption of the CYP reaction components based on hydrophobic interaction was also considered negligible. Therefore, the overall effect of the inks on the CYP activity could be assumed negligible.

The effect of surface charge characteristics was studied using two commonly used polyelectrolyte charge modifiers, cationic poly(diallyldimethylammonium chloride) (polyDADMAC) and anionic sodium polyacrylate (NaPA), and their impact on CYP2A6 model activity were determined by adding FCC modified with either compound to the enzyme incubation solution. As a result, polyDADMAC was shown to reduce the enzyme activity by half, whereas addition of NaPA resulted in about $40 \%$ increase in enzyme activity compared with the control (Figure 1a). On the basis of these results, it was concluded that the use of these surface charge modifiers should be avoided altogether or prevented from coming into contact 


\section{WILEY-VCH}

with the CYP enzyme due to uncertainty in the control of non-adsorbed polyelectrolyte concentration.

In addition to enzyme compatibility, the optical background of the FCC coatings was compared to that originating from a commercial filter paper (Whatman® Grade 1). On FCC, the lower limits of detection (LOD) and quantitation (LOQ) were determined for 7-hydroxycoumarin applied within printed containment ring patterns (inner diameter (i.d.) $5.0 \mathrm{~mm}, 2 \mathrm{~mm}$ wide barrier, volume uptake $1.0-1.8 \mu \mathrm{L}$ depending on the composition (Table S1)) defined by hydrophobic ink printed on the FCC coatings (Figure 1c). The calibration curves were established by applying 7-hydroxycoumarin onto the containment area (or filter paper). Sodium borate $(20 \mathrm{mM}, \mathrm{pH} 10.0)$ was used as the buffer to reach the maximum fluorescence quantum yield of 7-hydroxycoumarin, which is strongly $\mathrm{pH}$ dependent. ${ }^{[20]}$ Printing of similar ring patterns on filter paper was not feasible due to uncontrolled spreading of the AKD ink inside the ring patterns, rendering them completely hydrophobic. Therefore, the LOD and LOQ on filter paper were determined by applying the analyte directly onto the paper. To facilitate comparison of the LOD and LOQ levels between materials, the calibration curves were established as a function of the mole amount of the applied substance. The FCC coatings gave somewhat steeper slopes of calibration curve, indicating slightly better sensitivity compared with that of the filter paper (Figure 1b). LOD/LOQ were determined according to ICH guidelines $^{[21]}$ and were $0.5 / 1.4,0.4 / 1.4$ and $0.7 / 2.1$ pmol for 7-hydroxycoumarin applied on FCC with MFC A, FCC with MFC B and filter paper, respectively. The observed differences are likely due to the lower UV background originating from the FCC coating (3.0 \pm 0.5 a.u., FCC +5 pph MFC B, sampling number $n=36)$ compared with that of the filter paper $(6.6 \pm$ 0.5 a.u., $n=36$ ) (Figure $1 \mathrm{~b}$ ). On the basis of these results, it was concluded that FCC substrates provide a sensitive platform for the lateral flow assays, which is particularly important when 


\section{WILEY-VCH}

quantifying the trace-level amounts of drugs and their metabolites based on their inherent fluorescence in the UV range.

\subsection{Characterization of the wetting properties of the coating material}

To enhance the liquid wicking elongation capacity, the FCC-based coating formulations were optimized to maximize the pigment solids content and so to minimize the binder amount (quoted as parts by weight in respect to 100 parts by weight of FCC (pph)). The more the binder, the more porosity is reduced and, thus, the wetting and wicking capabilities of the coating are affected adversely. However, there must be sufficient amount of binder to bind the FCC particles to each other and to the base substrate to deliver a strong enough coated substrate which endures handling and printing.

In this study, two binder loadings, $5 \mathrm{pph}$ and $10 \mathrm{pph}$, for each binder (MFC A and B) were first characterized in terms of their porosity profiles. Figure 2a shows the first derivative of the cumulative mercury porosimetry intrusion curves of each of the four compositions. The data are presented in terms of specific pore volume, i.e. the pore volume in a given size range per unit weight of the sample. The main peaks in the pore size distribution between $1-3.1 \mu \mathrm{m}$ represent the interparticle pore volume, which contributes to permeability. The secondary peaks below $1 \mu \mathrm{m}$ represent the intraparticle pore size distributions, i.e. the internal pore volumes of the FCC particles, which ensure high capillarity. Both of these factors facilitate fast absorption of liquids within the coating. ${ }^{[2]}$ Figure 2 a clearly shows that MFC A at 10 pph fills the interparticle pores less than MFC B. MFC B exhibited higher binding power due to its greater degree of dispersed nanofibrilliated cellulose fines (Figure $2 b$ and c). Since the amount of binder also affects the volume of the interparticle pores of the coating (the more MFC is used, the less permeable the coating), we chose to use MFC B at 5 pph concentration as the binder in 


\section{WILEY-VCH}

this study to maintain the balance between maximizing the (sample) volume uptake and permeation wicking capability of the final assays whilst maintaining sufficient strength (see Table S2 for comparison of the interparticle specific pore volumes).

As illustrated in Figure 3, both volume uptake and evaporation rate can be altered by tuning the coating composition. The total volume uptake capacity of aqueous samples was in the range of $0.05-0.09 \mu \mathrm{L} . \mathrm{mm}^{-2}$ depending on the composition, with the highest volume uptake obtained with FCC containing 5 pph of MFC B, as expected (Figure 3a). Almost a two-fold increase in the volume uptake was reached adopting the MFC B at 5 pph versus MFC A at the strengthdemanding $10 \mathrm{pph}$. The differences observed between the theoretical and measured uptake volumes (Figure 3a) are likely related to both the binder's abilities to swell and absorb water, which results in deviation between the nominal and wetted volume occupancy in the coating, together with the observed spreading of AKD ink during print application, in turn reducing the inner diameter of the containment rings. On average, the ring cells stayed wetted for approximately 13 - $18 \mathrm{~min}$ at room temperature (Table S1). The evaporation rates were shown to be somewhat similar between the two binders at the lower 5 pph concentration, whereas increase in the binder amount seemed to slow down evaporation slightly, more so in case of MFC A than MFC B (Figure 3b). This confirms that evaporation is not related to bulk flow since the interparticle pore size, i.e. the permeability of the coating, is reduced (but not volume) in the case of MFC B when the binder level is increased. However, if we consider the surface area increase presented by the interparticle MFC matrix, seen as finer interparticle pores at the same pore volume (Figure 2c and Table S2), and that evaporation is diffusion and film flow driven rather than bulk flow driven, the reduction in bulk permeability is probably compensated for by the evaporative surface area increase. The ability of the MFC in question to swell and absorb water means that the binder itself, though solid in the porosimetry measurement, contributes to experimental effective porosity when wetted. 


\section{WILEY-VCH}

\subsection{Optimization of the Assay Design}

The final CYP assay was designed to comprise separate zones for enzyme reaction, separation (of the reaction components) and detection (of the metabolite), and an absorbent pad applied to the liquid feed reservoir to keep the assay wet for extended periods (up to $10 \mathrm{~min}$ ), even when heated to physiological temperature (Figure 4a). In addition to FCC, both binders, MFC A and B, were equally compatible with the enzymes (Figure 1a) and provided somewhat similar detection sensitivity in the UV range (Figure 1b). However, given the better wetting properties and binding power at low binder dosage when using MFC B, as described previously, FCC with MFC B (5 pph) was again chosen on these grounds as the coating formulation for further design development.

Although the FCC substrates remained wet for several minutes at room temperature, it was considered necessary, as mentioned above, to add a pre-wetted absorbent pad to the final assay design to be placed in contact with the reservoir to ensure that the reaction zone stays wet during the prolonged reaction times also at elevated temperature. Therefore, glass wool, pre-wetted with the buffer (0.1M Tris, $\mathrm{pH}$ 7.5) formed into an absorbent pad was incorporated together providing a constant supply of fresh buffer from the reservoir to the reaction zone. The duration of the enzyme incubation (reaction) period was adjusted to ensure that a sufficiently high amount of metabolite(s) is formed to exceed the LOQ of 7-hydroxycoumarin (ca. 1.4 pmol, Figure 1b).

After enzyme reaction, the assay was allowed to dry for $30 \mathrm{~min}$ prior to elution of the reaction components to the separation zone and eventually, the detection spot. The substrate of the chosen model reaction, coumarin 7-hydroxylation via CYP2A6, is non-fluorescent over the 


\section{WILEY-VCH}

entire $\mathrm{pH}$ range and so does not interfere with the detection of the fluorescent metabolite (7hydroxycoumarin). However, the cosubstrate (NADPH) has faint fluorescence in the near-UV range, which overlaps with the metabolite's fluorescence spectrum at neutral and basic $\mathrm{pH}$. Since NADPH is typically added to the CYP reaction solutions in excess, its concentration is easily ca. 100-fold greater than that of the formed metabolite, and thus NADPH complicates the fluorescence detection of the metabolite. In conventional CYP2A6 well-plate assays this can be overcome by acidifying the reaction solution prior to detection, typically with perchloric acid, which simultaneously denatures the enzyme and so terminates the reaction, after which the fluorescence excitation and emission maxima of NADPH and 7-hydroxycoumarin appear at different spectral wavelengths. ${ }^{[20]}$ However, acidification is not possible on the FCC-based lateral flow assay, because the acid would react with the carbonate fraction in FCC. Therefore, in-line separation from NADPH is required prior to detection of the metabolite. Conveniently, the coating material itself enables chromatographic separation of the chosen reaction components following the enzymatic reaction, as illustrated in Figure 4b. Chromatographic separation of the reaction components is also crucial in case the substrate and metabolite (of other CYP model reactions, for example) cannot be selectively separated based on their fluorescence properties alone, similarly to non-fluorescent coumarin and its fluorescent metabolite, 7-hydroxycoumarin.

To facilitate online chromatographic separation upon elution of the reaction solution from the reaction zone to the detection spot, a 3-mm-wide and 15-mm-long separation channel was patterned into the final design. The assay and channel dimensions were outlined by full barriers using two inkjet applied AKD ink layers $\left(96.5 \pm 7.0 \mathrm{~cm}^{3} \mathrm{~m}^{-2}\right) .{ }^{[12 \mathrm{f}]}$ The channel is separated from the reaction zone by a semi-barrier (Figure 4a). The semi-barrier retains the reaction solution in the designated zone while the reaction is running, liquid being fed by the absorbent pad used as the fresh buffer storage, but afterwards allows elution of the reaction components upon 


\section{WILEY-VCH}

application of the elution buffer (20 mM sodium borate, $\mathrm{pH} \mathrm{10).} \mathrm{The} \mathrm{semi-permeable} \mathrm{fluid}$ barrier between the reaction and separation zones was inkjet printed using polystyrene (PS) ink, which is known to require many more application layers to become fully water-tight, and thus provides for precise control of permeability according to the number of layers applied. ${ }^{[12 f]}$ Four layers of PS ink was found to work satisfactorily to prevent liquid from spreading into the separation channel during the reaction with the absorbent moistening pad in place, whilst allowing the liquid to flow through upon application of the elution buffer.

With the final assay design (Figure 4a), $25 \mu \mathrm{L}$ of the elution buffer was required to induce high enough wicking force to overcome the repulsion forces of the semi-barrier and to ensure full wetting of the separation zone until the arrow-shaped detection spot. The length of the separation channel determines the total wetted area, and, thus, the required buffer volume, which eventually affects the elution time. Based on the material specific volume uptake data (Figure 2a), the theoretical volume required to wet the final assay (here, $142 \mathrm{~mm}^{2}$ ) was $13 \mu \mathrm{L}$, which is in line with the experimentally determined elution volume $(25 \mu \mathrm{L})$, taking the evaporation into consideration. If needed, larger elution volumes can also be used for wetting an even longer separation path (larger areas), thus increasing the overall chromatographic resolving power. In this work, however, a relatively short separation path was favored because it allowed for shorter elution times (ca. $5 \mathrm{~min}$ ) and the chromatographic separation of the metabolite from NADPH was obtained easily, as described below. It was also shown that the capillary wicking kinetics of the final assay design were well repeatable (Figure 4c), including the slow down effect by the semi-barrier.

To characterize the chromatographic behavior of the coating formulation, thin layer chromatography (TLC) experiments were performed independently to optimize the conditions for separation of NADPH and 7-hydroxycoumarin. For this purpose, glass slides were coated 


\section{WILEY-VCH}

with FCC containing 5 pph MFC B and NADPH (1 nmol) and 7-hydroxycoumarin (0.5 nmol) were applied to the FCC substrate in the incubation buffer (0.1M Tris, $\mathrm{pH} 7.5)$ and eluted with the elution buffer (20 mM sodium borate, pH 10) (Figure S1). It was observed that 7hydroxycoumarin eluted along with the solvent front with an $R_{\mathrm{f}}$ value of ca. 1 , whereas the anionic NADPH was significantly retained by the FCC (Figure 4d). The surface of FCC consists of pores built from protruding hydroxyapatite platelet structures on a calcium carbonate particulate core, and, thus, while the particle pores are anionic, the edges of the platelets have a slight cationic charge ${ }^{[23]}$ capable of interacting with the anionic NADPH. To investigate further the impact of surface charge compared with the inherent zwitterionic nature of FCC, either anionic (sodium polyacrylate, NaPA) or cationic (polyDADMAC) polyelectrolyte solution was applied on FCC by inkjet printing to neutralize the inherent complementary charge. ${ }^{[24]}$ As expected, the chromatographic behavior of NADPH and 7-hydroxycoumarin when applied onto the cationic treatment was similar to that on bare FCC (Figure 4d). When the compounds were applied on the anionically treated surface, NADPH was no longer retained based on strong electrostatic interaction, but only by weak hydrophilic interaction, and thus eluted closer to the solvent front with $R_{\mathrm{f}}$ of ca. 0.75 (Figure $4 \mathrm{~d}$ ). The possibility to tune the inherent surface chemistry of FCC locally via inkjet printing of cationic or anionic treatments further increases the selectivity of separation, although care should be taken, as mentioned earlier, not to include the treatments at the point of enzymatic reaction due to their potential inhibitory or enhancing effects on the enzyme activity (Figure 1a). In this work, bare FCC was found sufficient to provide chromatographic separation of the reaction components, and so charge addition was not further pursued. On the final design, the obtained $R_{\mathrm{f}}$ values for NADPH and 7-hydroxycoumarin were $0.23 \pm 0.01$ and $0.98 \pm 0.03$, respectively. When in excess compared with the FCC cationic adsorption capacity, NADPH is not fully adsorbed at the reaction zone, maintaining its reactivity, but will be retained by the coating during wicking in the separation channel even if penetrating the semi-barrier. 


\section{WILEY-VCH}

Finally, the geometry of the detection spot was also optimized. Instead of using the roundshaped end (similar to ring patterns), an arrowhead shape was adopted to the final design. This was to avoid the Marangoni effect (coffee stain ring), which is characteristic to ring patterns and results in concentration of the analytes around the edges. Instead, the arrowhead shape enables concentration of the sample into a well-defined area at the tip. However, depending on the detection window size and shape, the detected area may also partly cover the AKD ink barrier, which may induce significant background due to its autofluorescence. In this work, by carefully selecting the dye used for visualizing the hydrophobic barriers, we were able to eliminate the AKD-related background as the blue dyed layer formed on and between the coating pigment particles effectively scattered the UV light (at the excitation wavelengths used, $355 \pm 5 \mathrm{~nm}$ ), with no emission at the desired emission wavelength range (in the detected emission wavelength range of $400-700 \mathrm{~nm}$ ). Some portions of the fluorescence readings nonetheless may originate from the AKD ink, because the AKD spreads more than the dye on the coating. Therefore, fluorescence was recorded before and after elution for all arrowhead tips, and the background (before) was subtracted from the final values (after) case-by-case.

\subsection{Validation of the lateral flow CYP2A6 assays}

Once the microfluidic design was optimized, the detection sensitivity of the final assay was determined according to ICH guidelines. ${ }^{[21]}$ Before fluorescence detection, the desired amounts of metabolite were applied onto the reaction zones in $1.5 \mu \mathrm{L}$ volumes of $0.1 \mathrm{M}$ Tris buffer $(\mathrm{pH}$ 7.5), allowed to dry, and eluted to the end of the separation channel with $25 \mu \mathrm{L}$ of $20 \mathrm{mM}$ sodium borate buffer ( $\mathrm{pH}$ 10). To account for all possible background interference originating from the reaction components, both NADPH $(1.5 \mathrm{nmol})$ and HLM $(1.5 \mu \mathrm{g})$ were also applied on each assay prior to addition of 7-hydroxycoumarin. In addition, the assay was heated to 37 


\section{WILEY-VCH}

${ }^{\circ} \mathrm{C}$ before elution to include the effects of possible heat degradation products (of NADPH, for example). Under these conditions, the obtained LOD and LOQ were 0.9 and 2.7 pmol, respectively, which were very well in line with those obtained with the ring patterns (Figure 1b), indicating that no significant loss of sample takes place during the elution (separation) step. The linearity of the assay was also sufficiently good $\left(R^{2}=0.9554\right)$ between 0.38 and 3.00 pmol (Figure 5a).

Finally, CYP2A6 enzyme reactions were carried out on the developed lateral flow assays to examine their feasibility for preclinical in vitro drug-drug or drug-chemical interaction screening. In conventional end-point assays, the reaction is typically initiated by the addition of the cosubstrate and terminated by the addition of the acid or organic solvent, which precipitates the proteins. In this work, the reaction was terminated by removing the absorbent pad at a fixed time point $(t=10 \mathrm{~min})$, which resulted in denaturation of the proteins due to drying. The lateral flow enzyme reactions were carried out by applying the cosubstrate (NADPH, $1.5 \mathrm{nmol}$ in deionized (DI) water) and the substrate (coumarin, 75 pmol in DI water) onto the reaction zone, allowing drying between applications, after which the pre-wetted absorbent pad was placed over the reservoir and HLM (1.5 - $15 \mu \mathrm{g}$ in $0.1 \mathrm{M}$ Tris buffer, $\mathrm{pH}$ 7.5) was applied to the reaction zone to initiate the reaction. The assay was heated to $37^{\circ} \mathrm{C}$ prior to addition of any further reagents, and kept wet for 10 min by adding more buffer to the absorbent pad, if needed. After the 10 min reaction period, the absorbent pad was removed and the assay allowed to dry. The reaction products were then eluted to the separation channel with $25 \mu \mathrm{L}$ of the $20 \mathrm{mM}$ sodium borate ( $\mathrm{pH} 10.0$ ) buffer, as described earlier, so as to allow simultaneous $\mathrm{pH}$ shift from the neutral reaction $\mathrm{pH}$ of 7.5 to basic detection $\mathrm{pH}$ of 10.0 (providing maximum quantum yield for umbelliferone at ex $355 \mathrm{~nm}$ ). 


\section{WILEY-VCH}

To be able to determine the critical enzyme inhibition parameters from observed interactions, i.e., at half maximum inhibition $\mathrm{IC}_{50}$ or inhibitory constant $K_{\mathrm{i}}$, it is important to ensure that the reaction kinetics is linearly dependent on the enzyme amount and the reaction time. Here, the metabolite production rate $\left(\mathrm{pmol} \cdot \mathrm{min}^{-1}\right)$ increased linearly as a function of enzyme concentration between 1.5 and $15 \mu \mathrm{g}$ total protein concentration (HLM) $\left(R^{2}=0.9520\right)$ (Figure 5b). At the lowest total protein concentrations, however, the amount of metabolite formed was so small that the inherent deviation in the biological enzyme activity, combined with the precision of the reagent dosing, overpowered the metabolite signal and resulted in relatively large variation between the parallel assays $(n=4$ at each total protein concentration level, Figure $5 b)$. However, a statistically significant difference $(p=0.032)$ to the negative control reactions without the enzyme ( $\left.44 \pm 15 \mathrm{fmol} \cdot \mathrm{min}^{-1}, n=4\right)$ was obtained until at total protein concentration of $12 \mu \mathrm{g}\left(134 \pm 53 \mathrm{fmol} \cdot \mathrm{min}^{-1}, n=4\right)$. This concentration was still in the linear range suggesting feasibility of the assay for the envisioned enzyme inhibition screening. In all, these results suggest that the developed lateral flow CYP assay might serve as a rapid and low-cost interaction screening assay.

\section{Conclusions}

A microfluidic lateral flow assay to study human phase I metabolism interactions mediated by cytochrome P450 (CYP) enzymes was developed. The lateral flow assay is constructed from a specialty porous particle coating (FCC) and MFC binder. An inkjet-printed hydrophobic barrier pattern, including both fully and semi-impermeable components, is fabricated on the coating using AKD and PS inks, respectively. The assay consists of three zones, each for the enzymatic reaction, the separation of the reaction products and the detection of the formed metabolite. These are supplemented by a buffer reservoir zone and an absorbent pad to facilitate constant wetting of the substrate for prolonged reaction times even at elevated temperatures (here, 37 


\section{WILEY-VCH}

$\left.{ }^{\circ} \mathrm{C}\right)$. The design also compises a semi-barrier to prevent the reaction solution from escaping to the separation zone during the enzymatic reaction. The properties of the coating enable chromatographic separation of the reaction components and, thus, selective transport of the metabolite to the detection zone, where the metabolite can be quantified based on UV fluorescence. Conveniently, sensitive detection of the chosen model compounds is facilitated by the fact that the coating material in itself shows no significant background intereference (autofluorescence).

To our knowledge, the concept of microfluidic, inkjet-printed lateral flow assays has not been applied to CYP metabolism research until the present work. Our proof-of-concept assays show that the FCC-based platform coupled with fluorescence detection is a simple and viable method for studying CYP2A6 mediated reactions in vitro, and, thus, provides a convenient screening tool to examine the drug-drug or drug-chemical interactions at comparatively low cost compared with the conventional well-plate assays. The reduced consumption of biochemicals and lower amount of waste not only decrease the total costs, but have added value as such toward sustainable development making use of sustainable raw materials. In addition to lower cost, the possibility for sample manipulation (e.g., separation of reaction components) via chromatographic interaction - enforced by selective surface modifications, if needed - provides a clear advantage over conventional well-plate assays.

\section{Experimental Section}

Coating Preparation: A highly porous form of pharmaceutical grade FCC, provided by Omya International AG, Oftringen, Switzerland, in which the functionalization consists of a shell-core structure of hydroxyapatite $(52.7 \%)$ and calcium carbonate $(47.3 \%)$, was used as a coating pigment. Two types of MFC, a commercially available Arbocel MF-40-7 (J. Rettenmaier \& 


\section{WILEY-VCH}

Söhne GmbH + Co KG, Rosenberg, Germany), termed MFC A, and a mechanically produced micro nanofibrillated cellulose (Omya International AG, Oftringen, Switzerland), termed MFC B, were evaluated as binders. SuperYUPO® (Yupo Corporation, Tokyo, Japan), pigment filled polypropylene film (thickness $80 \mu \mathrm{m}$ ), was used as the inert base substrate for the coatings. Coating colors were applied with a K202 Control Coater (RK PrintCoat Instruments Ltd., Litlington, UK.) employing two spirally wound rods, applying a $150 \mu \mathrm{m}$ (MFC A containing) and a $400 \mu \mathrm{m}$ (MFC B containing) thick coating, respectively, as a wet layer with a speed setting of $6 \mathrm{~m} \cdot \mathrm{min}^{-1}$. The coatings were dried in laboratory atmosphere.

Coating Characterization: Solids content was determined with a Precisa HA 300 Moisture Balance (Precisa Gravimetrics AG, Dietikon, Switzerland). Viscosity was measured using a Brookfield DV-II+ viscometer (Brookfield Engineering Laboratories, Inc., Massachusetts, USA) using spindle \#4 and a rotation speed of $100 \mathrm{~min}^{-1}$. Coat weight was calculated by measuring the weight of pieces of the coated polypropylene film $\left(5 \times 5 \mathrm{~cm}^{2}\right)$, using a Mettler AE260 analytical balance (Mettler-Toledo International Inc., Columbus, Ohio, USA), deducting the weight of the film and dividing with the area of the sample $(n=5)$. Coating thickness was measured with an SE250D micrometer (Lorentzen \& Wettre, Kista, Sweden), having a $2 \mathrm{~cm}^{2}$ measurement area spindle and measurement pressure of $100 \mathrm{kPa}(n=10)$. The dry coating thickness was determined from the measured coated films by subtracting the nominal thickness of the polypropylene film. Scanning electron micrographs (SEM) were obtained with a Sigma VP field emission scanning electron microscope (Carl Zeiss AG, Oberkochen, Germany). Coating pore volume was measured with an Autopore IV mercury intrusion porosimeter (Micromeritics, Norcross, USA) from coating samples dried in a petri dish (diameter $5.5 \mathrm{~cm}$ ) in normal laboratory conditions. Table $\mathbf{S 3}$ shows the properties of the coating formulations. The measured uptake volumes were experimentally determined by applying different volumes of Tris buffer into the center of the ring cell (i.d. $=5 \mathrm{~mm}$ ) until a 


\section{WILEY-VCH}

reflective liquid layer remaining on the surface was just apparent. The theoretical total volume uptake was calculated by multiplying the internal area of the circular ring cell, the coat weight of the given coating and the corresponding specific pore volume data (Table S1). Evaporation experiments were performed by applying Tris buffer $(1.0-1.8 \mu \mathrm{L} ; 0.1 \mathrm{M}, \mathrm{pH} 7.5)$ with $\mathrm{MgCl}_{2}$ (3.5 $\mathrm{mM}$ ) onto the central area of inkjet-printed rings (or on filter paper) and measuring the change of mass as a function of time with a Mettler Toledo MT5 microbalance coupled with Mettler Toledo Balance Link - DSC software (Mettler-Toledo International Inc., Columbus, Ohio, USA) $(n=3)$.

Functional Ink and Printing of Patterns: Two functional inks, using two different hydrophobizing agents, were used to fabricate patterns onto the coated samples. ${ }^{[11 \mathrm{e}, \mathrm{f}]}$ The inks were formulated using polystyrene PS (Sigma-Aldrich, St. Louis, USA) and alkyl ketene dimer AKD (BASF, Ludwigshafen, Germany), dissolved in p-xylene solvent (VWR, Vienna, Austria). The PS ink was colored with Sudan Red G (Sigma-Aldrich, St. Louis, USA), and the AKD ink was colored with Blue 807 dye (Kremer Pigmente GmbH, \& Co. KG, Aichstetten, Germany) as markers. Both inks were printed using $10 \mu \mathrm{m}$ drop spacing using a DMP-2831 research inkjet printer (Fujifilm Dimatix, Santa Clara, USA) employing DMC-11610 ink cartridges with 10 pL nominal drop volume. Two polyelectrolyte-based inks, cationic (polyDADMAC) and anionic (sodium polyacrylate NaPA) were used to modify the surface charge of the coating. ${ }^{[22]}$ The polyDADMAC ink was printed using $15 \mu \mathrm{m}$ drop spacing (6 layers) and $\mathrm{NaPa}$ ink using $12 \mu \mathrm{m}$ drop spacing (6 layers).

Reagents used in Lateral Flow Enzyme Assays: Human liver microsomes (HLM) were purchased from BD Biosciences (Woburn, MA, USA). Coumarin, 7-hydroxycoumarin (umbelliferone), $\beta$-Nicotinamide adenine dinucleotide 2'-phosphate reduced tetrasodium salt hydrate (NADPH), dimethyl sulfoxide (DMSO) and Trizma base were purchased from Sigma- 


\section{WILEY-VCH}

Aldrich (St. Louis, MO, USA). Magnesium chloride $\left(\mathrm{MgCl}_{2}\right)$, boric acid, hydrochloric acid, and perchloric acid $\left(\mathrm{HClO}_{4}\right)$ were from Riedel-de-Haën (Seelze, Germany). All reagents and solvents were of analytical or HPLC grade. Water was purified with a Milli-Q water purification system (Millipore, Molsheim, France).

Characterization of the materials-enzyme compatibility: FCC, the binders MFC A and B, and the polyelectrolytes NaPA and polyDADMAC were individually screened for their potential effect on the selected model reaction, i.e. CYP2A6-mediated coumarin 7-hydroxylation. These reactions were carried out in a total volume of $100 \mu \mathrm{L}$ of Tris buffer $(0.1 \mathrm{M}, 3.5 \mathrm{mM} \mathrm{MgCl}$, $\mathrm{pH} 7.5)$ in the presence of HLM $\left(0.4 \mathrm{mg} \cdot \mathrm{mL}^{-1}\right)$, NADPH $(1 \mathrm{mM})$ and coumarin $(4 \mu \mathrm{M})$. Respectively, FCC (20 w/w\%), MFC A (6.32 w/w\%) and MFC B (1.32 w/w\%) was added to the reaction mixture as aqueous suspension prior to dilution to the final reaction volume. The $\mathrm{NaPA}$ and polyDADMAC were added to the reaction mixture together with the FCC using 0.5 pph concentration, each with respect to solid content of $26 \mathrm{w} / \mathrm{w} \%$ and $24 \mathrm{w} / \mathrm{w} \% \mathrm{FCC}$, respectively. The enzyme reactions were carried out in Eppendorf tubes and initiated by the addition of $\mathrm{NADPH}$, incubated for $15 \mathrm{~min}$ at $37{ }^{\circ} \mathrm{C}$ and terminated with the addition of perchloric acid $(10 \mu \mathrm{L}, 4 \mathrm{M})$. The reaction solutions were put on ice for about 10 min to allow the proteins to precipitate, after which they were centrifuged for $10 \mathrm{~min}$ at $16000 \mathrm{~g}$. The supernatants were then separated for analysis on a Varioskan ${ }^{\circledR}$ Flash well-plate reader (Thermo Scientific, Vantaa, Finland). The amount of 7-hydroxycoumarin was quantified using fluorescence detection (excitation $325 \mathrm{~nm}$, emission $470 \mathrm{~nm}$ ) and the CYP2A6 activities were compared between control reactions (no added materials) and reactions carried out in the presence of FCC, MFC A, MFC B, NaPA or polyDADMAC.

Fluorescence Microscopy (Lateral Flow Assays): Fluorescence detection was accomplished with a Zeiss Axioscope A1 upright epifluorescence microscope (Carl Zeiss Oy, Espoo, Finland) 


\section{WILEY-VCH}

equipped with a 10× objective and a broad-band halogen lamp (HAL100W) with a $355 \pm 10$ $\mathrm{nm}$ filter as excitation source. The fluorescence emission was collected over the range $400-$ $700 \mathrm{~nm}$ using a Hamamatsu R5929 photomultiplier tube equipped with integrated housing and a signal amplifier module (Cairn Research). Coated platforms and a qualitative filter paper (Whatman® Grade 1) purchased from Sigma-Aldrich (St. Louis, MO, USA) were used as substrates.

Thin Layer Chromatography (TLC): The chromatographic separation of the co-substrate, $\mathrm{NADPH}$, and the metabolite, 7-hydroxycoumarin, on the FCC coating was investigated using both coated microscope glass slides $\left(25 \times 75 \mathrm{~mm}^{2}\right.$, VWR International) and the final assay design (FCC +5 pph MFC B). In the case of coated glass slides, NADPH $(1 \mu \mathrm{L}, 1 \mathrm{mM})$ and umbelliferone $(1 \mu \mathrm{L}, 500 \mu \mathrm{M})$ were pipetted onto the plain FCC +5 pph MFC B coating and onto inkjet-printed polyDADMAC or NaPA areas $\left(1 \times 2.5 \mathrm{~cm}^{2}\right)$ on the coating (Figure S1), and the samples were placed into a chromatographic chamber containing sodium borate buffer (20 mM, pH 10). The samples were examined under UV light using a MiniMatcher MM-4e (GTI Graphic Technology Inc., New York, USA) equipped with a UV lamp (Philips TL 8W/08 F8 T5/BLB $365 \mathrm{~nm}$ ) before and after the experiment. Chromatographic separation on the final assay design (FCC +5 pph MFC B) was tested by pipetting NADPH $(1.5 \mu \mathrm{L}, 1 \mathrm{mM})$ and umbelliferone $(1.5 \mu \mathrm{L}, 5 \mu \mathrm{M})$ to the reaction zone and eluting the sample with the sodium borate buffer solution $(25 \mu \mathrm{L})$ in a horizontal orientation. The sample was examined under UV light before and after elution. The retention factors $\left(R_{\mathrm{f}}\right)$ were calculated by dividing the distance travelled by the compound of interest with the distance travelled by the solvent front.

Lateral flow enzyme assays: The background intensity of the sample was measured by prewetting the tip of the arrowhead detection zone with the sodium borate buffer $(0.5 \mu \mathrm{L})$. The sample was placed on a digital hot plate (model 062, Labotect) at $37^{\circ} \mathrm{C}$ and allowed to warm 


\section{WILEY-VCH}

up. NADPH $(1 \mathrm{mM})$ and coumarin $(50 \mu \mathrm{M})$ in DI water $(1.5 \mu \mathrm{L})$ was pipetted to the reaction zone and allowed to dry. Absorbent pad (Whatman® glass microfiber filter, Grade GF/F, GE Healthcare Europe $\mathrm{GmbH}$, Finland) pre-wetted with Tris buffer (25 $\mu \mathrm{L}, 0.1 \mathrm{M}, \mathrm{pH} 7.5)$ containing $\mathrm{MgCl}_{2}(3.5 \mathrm{mM})$ was applied to the absorbent pad zone to keep the reaction zone wetted. HLM in the Tris buffer $\left(1.5 \mu \mathrm{L}, 0-1-2-4-6-8-10 \mathrm{mg} \cdot \mathrm{mL}^{-1}\right.$ total protein concentration) was applied to the reaction zone and more Tris buffer was dispensed to the absorbent pad, as needed, during the 10 min experiment to ensure that the reaction zone stayed wet over the entire reaction period. Negative controls were performed without HLM and without NADPH and coumarin. After 10 min the absorbent pad was removed and the sample was allowed to dry. The metabolite was eluted to the detection zone by adding sodium borate buffer $(20 \mathrm{mM}, \mathrm{pH}$ $10,25 \mu \mathrm{L}$ ) to the moistening zone. The sample was then allowed to dry for at least $30 \mathrm{~min}$. Fluorescence detection was carried out by re-wetting the tip with the sodium borate buffer (4 $\mu L)$.

\section{Supporting Information}

Supporting Information is available from the Wiley Online Library or from the author.

\section{Acknowledgements}

Funding for the project was supported by Omya International AG (Jutila and Koivunen, Aalto University), the European Research Council (project no. 311705/CUMTAS, Sikanen, University of Helsinki), the Academy of Finland (project no. 304400), and the Doctoral Program in Drug Research (Kiiski, University of Helsinki). Silvan Fischer (Omya International $\mathrm{AG}$ ) is acknowledged for the SEM analysis, Cathy Ridgway (Omya International AG) for porosimetry and Melanie Kolb (Omya International AG) for determining pigment mineral content.

Received: ((will be filled in by the editorial staff))

Revised: ((will be filled in by the editorial staff)) Published online: ((will be filled in by the editorial staff))

\section{References}

[1] M. Ruokolainen, T. Gul, H. Permentier, T. Sikanen, R. Kostiainen, T. Kotiaho, Eur. J. Pharm. Sci. 2016, 83, 36. 


\section{WILEY-VCH}

[2] a) F.P. Guengerich, Chem. Res. Toxicol. 2008, 21, 70; b) F.P. Guengerich, in Metabolism of Drugs and Other Xenobiotics (Eds. P. Anzenbacher, U.M. Zanger), Wiley-VCH, Weinheim, Germany 2012, Ch. 2.

[3] E. Hodqson, R.L. Rose, J. Biochem. Mol. Toxicol. 2007, 21, 182.

[4] a) D.M. Cate, J.A. Adkins, J. Mettakoonpitak, C.S. Henry, Anal. Chem. 2015, 87, 19; b) S.F. Cheung, S.K.L. Cheng, D.T. Kamei, J. Lab. Autom. 2015, 20, 316; c) M. Sajid, A-N. Kawde, M. Daud, J. Saudi Chem. Soc. 2015, 19, 689, d) N.A. Meredith, C. Quinn, D.M. Cate, T.H. Reilly III, J. Volckens, C.S. Henry, Analyst 2016, 141, 1874; e) K. Yamada, H. Shibata, K. Suzuki, D. Citterio, Lab Chip 2017, 17, 1206.

[5] a) A. W. Martinez, S. T. Phillips, M. J. Butte, G. M. Whitesides, Angew. Chem. 2007, 119, 1340; Angew. Chem. Int. Ed. 2007, 46, 1318; b) A.W. Martinez, S.T. Phillips, G. Whitesides, Anal. Chem. 2010, 82, 3.

[6] a) Q. He, C. Ma, X. Hu, H. Chen, Anal. Chem. 2013, 85, 1327; b) H. Asano, Y. Shiraishi, Anal. Chim. Acta. 2015, 883, 55; c) L. Cao, C. Fang, R. Zeng, X. Zhao, Y. Jiang, Z. Chen, Biosens. Bioelectron. 2017, 92, 87.

[7] a) X. Li, J. Tian, T. Nguen, W. Shen, Anal. Chem. 2008, 80, 9131; b) P. Kao, C. Hsu, Microfluid. Nanofluid. 2014, 16, 811.

[8] G. Chitnis, Z. Ding, C. Chang, C. A. Savran, B. Ziaie, Lab Chip 2011, 11, 1161.

[9] a) E. Carrilho, A.W. Martinez, G.M. Whitesides, Anal. Chem. 2009, 81, 7091; b) D.

Sechi, B. Greer, J. Johnson, N. Hashemi, Anal. Chem. 2013, 85, 10733; c) C. Renault, J. Koehne, A.J. Ricco, R.M. Crooks, Langmuir 2014, 30, 7030.

[10] a) W. Dungchai, O. Chailapakul, C.S. Henry, Analyst 2011, 136, 77; b) Y. Sameenoi, P. N. Nongkai, S. Nouanthavong, C. S. Henry, D. Nacapricha, Analyst 2014, 139, 6580.

[11] a) J. Olkkonen, K. Lehtinen, T. Erho, Anal. Chem. 2010, 82, 10246; b) A. Määttänen, D. Fors, S. Wang, D. Valtakari, P. Ihalainen, J. Peltonen, Sens. Actuators B 2011, 160, 1404. 


\section{WILEY-VCH}

[12] a) K. Abe, K. Suzuki, D. Citterio, Anal. Chem. 2008, 80, 6928; b) X. Li, J. Tian, G. Garnier, W. Shen, Colloids Surf. B 2010, 76, 564; c) K. Maejima, S. Tomikawa, K. Suzuki, D. Citterio, $R S C A d v$. 2013, 3, 9258; d) V. Rajendra, C. Sicard, J. D. Brennan, M. A. Brook, Analyst 2014, 139, 6361; e) R. Koivunen, E. Jutila, P. Gane, JPMTR 2015, 4, 7; f) R. Koivunen, E. Jutila, R. Bollström, P. Gane, Microfluid. Nanofluid. 2016, 20:83.

[13] G. Jenkins, Y. Wang, Y. L. Xie, Q. Wu, W. Huang, L. Wang, X. Yang, Microfluid. Nanofluid. 2015, 19, 251.

[14] a) X. Li, D.R. Ballerini, W. Shen, Biomicrofluidics 2012, 6, 011301, b) A.K. Yetisen, M.S. Akram, C.R. Lowe, Lab Chip 2013, 13, 2210; c) K. Yamada, T.G. Henares, K. Suzuki, D. Citterio, Angew. Chem. 2015, 127, 5384; Angew. Chem. Int. Ed. Engl. 2015, 54, 5294.

[15] Z.W. Zhong, Z.P. Wang, G.X.D Huang, Microsyst. Technol. 2012, 18, 649.

[16] Y. Yang, E. Noviana, M.P. Nguyen, B.J. Geiss, D.S. Dandy, C.S. Henry, Anal. Chem. 2017, 89, 71.

[17] a) C-M. Cheng, A.W. Martinez, J. Gong, C.R. Mace, S.T. Phillips, E. Carrilho, K.A. Mirica, G.M. Whitesides, Angew. Chem. 2010, 122, 4881; Angew. Chem. Int. Ed. 2010, 49, 4771; b) A. Apilux, Y. Ukita, M. Chikae, O. Chailapakul, Y. Takamura, Chip Lab 2013, 13, 126; c) R.C. Murdock, L. Shen, D.K. Griffin, N. Kelley-Loughnane, I. Papautsky, J.A. Hagen, Anal. Chem. 2013, 85, 11634; d) S.K. Chan, T.S. Lim, Anal. Methods 2016, 8, 1431.

[18] a) J. Songok, M. Tuominen, H. Teisala, J. Haapanen, J. Mäkelä, J. Kuusipalo, M. Toivakka, ACS Appl. Mater. Interfaces 2014, 6, 20060; b) J. Songok, M. Toivakka, Microfluid. Nanofluid. 2016, 20:63.

[19] A. Rossini, T. de Almeida Simão, R.M. Albano, L.F. Pinto, Pharmacogenomics 2008, 9, 1737.

[20] D.W. Fink, W.R. Koehler, Anal. Chem. 1970, 42, 990. 


\section{WILEY-VCH}

[21] International Conference on Harmonization of Technical Requirements for Registration of

Pharmaceuticals for Human Use. Validation of Analytical Procedures: Text and Methodology,

Q2 (R1), 2005.

[22] a) C. Ridgway, P.A.C. Gane, J. Schoelkopf, Colloids Surf. A 2004, 236, 91; b) C.

Ridgway, P.A.C. Gane, J. Schoelkopf, Transp. Porous Media 2006, 63, 239.

[23] T. Lamminmäki, J.P. Kettle, P.A.C. Gane, in $8^{\text {th }}$ International Paper and Coating

Chemistry Symposium, Stockholm, Sweden 2012, p. 81.

[24] R. Koivunen, E. Jutila, R. Bollström, P. Gane, Colloids Surf. A 2017, 522, 218.
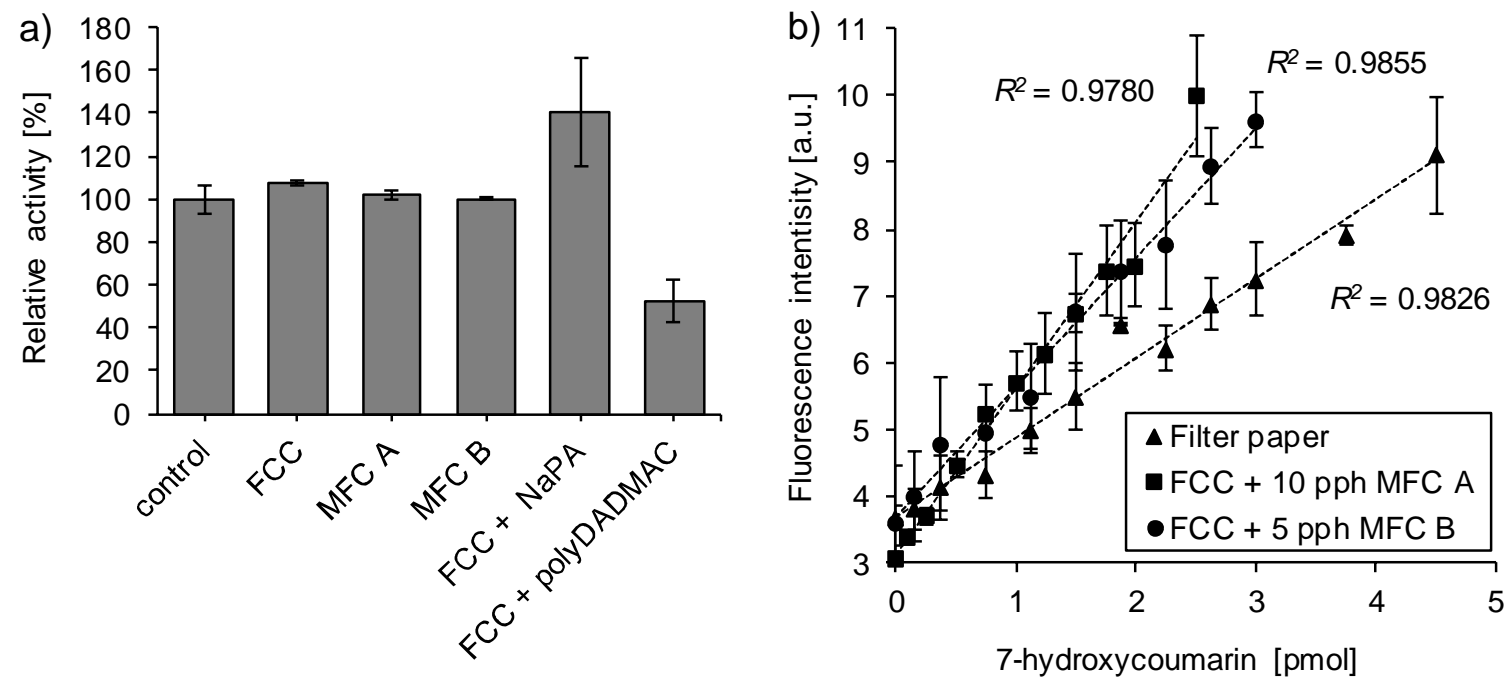

c)

Figure 1. a) The influence of the coating pigment (FCC), the binders (MFC A and MFC B) and the charge modifiers (NaPA and polyDADMAC) on the CYP2A6 activity. b) Comparison of the calibration curves determined for 7-hydroxycoumarin using the two different FCC-based coatings or the commercial filter paper. The error bars represent deviation between repeated measurements $(n=3)$. c) Inkjet-printed ring patterns used in determination of the optical background from coated substrates. 


\section{WILEY-VCH}
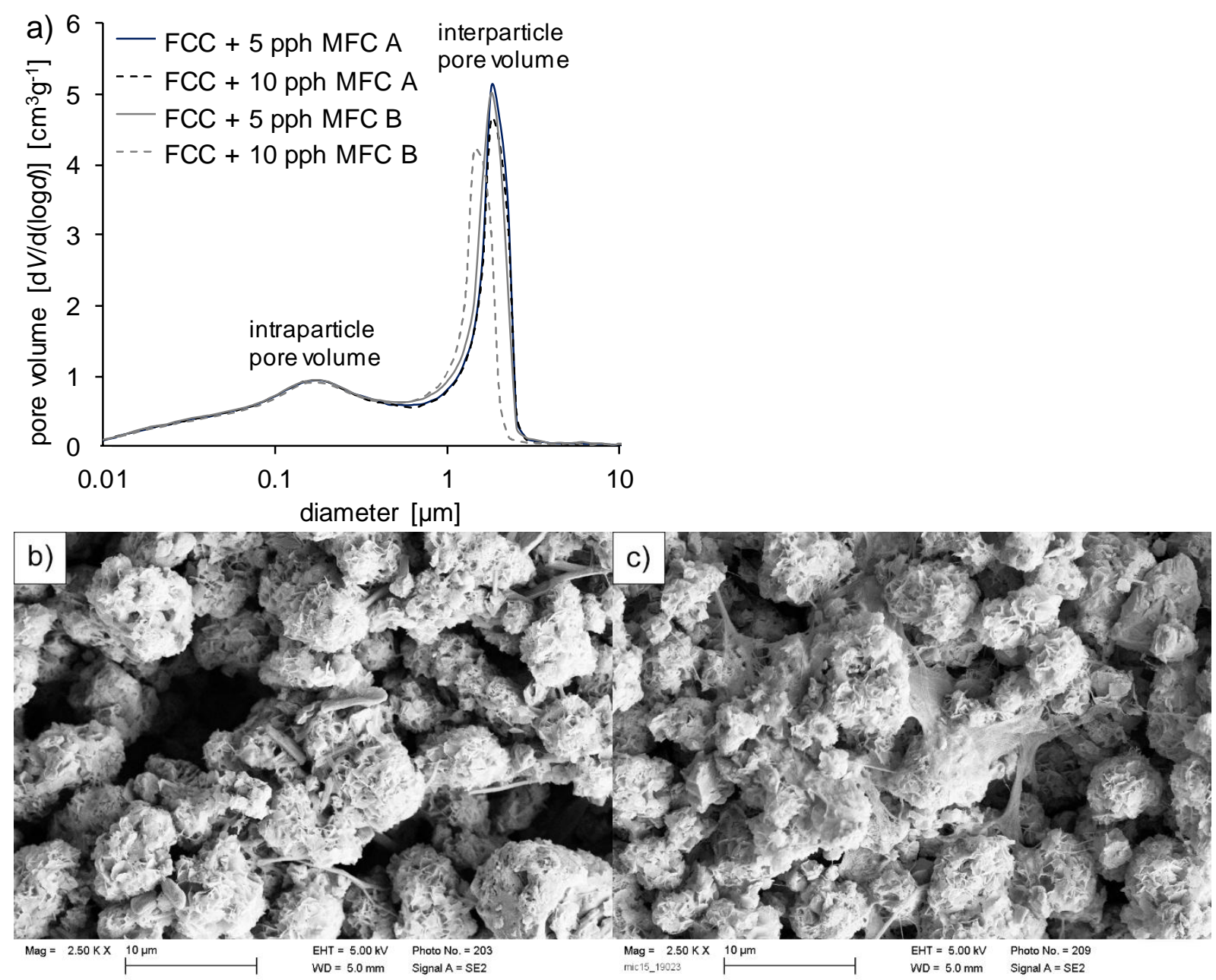

Figure 2. a) Pore size distribution of the different coating formulations tested, showing the significant reduction in interparticle specific pore volume, which controls permeability, on increasing addition of binder (note: MFC B also reduces the interparticle pore size), and scanning electron microscope (SEM) images of FCC particles combined with b) MFC A (10 pph), and c) MFC B (5 pph).
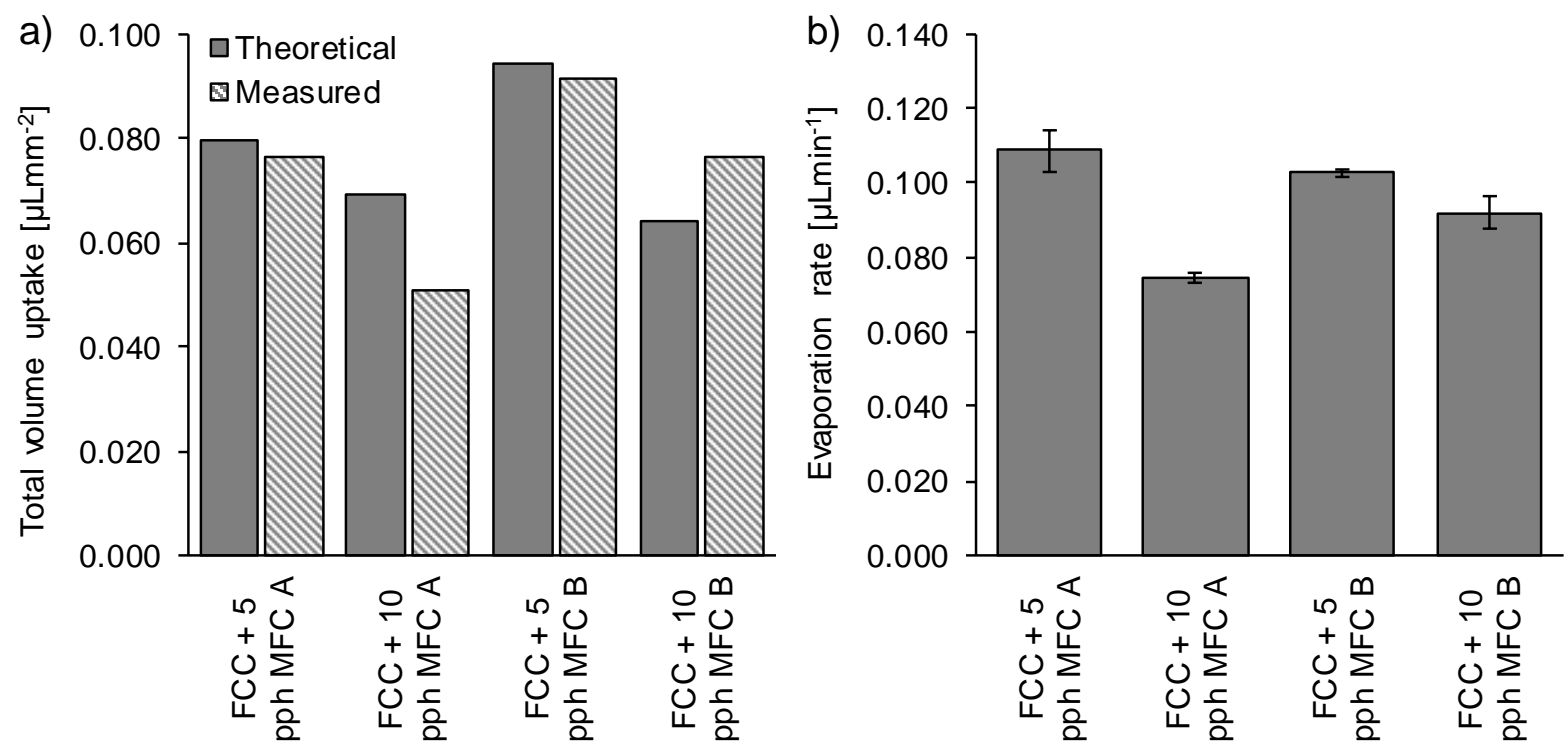


\section{WILEY-VCH}

Figure 3. a) Comparison of the theoretical and measured volume uptake of the different coating compositions tested. b) Comparison of the evaporation rate of the different coating compositions tested - the error bars represent deviation between repeated measurements $(n=$ 3 ) - to illustrate the enhanced evaporation rate obtained on the assay, a lower evaporation rate of $0.04 \pm 0.00 \mu \mathrm{L} . \mathrm{min}^{-1}$ was obtained for $1 \mu \mathrm{L}$ of the aqueous buffer $(0.1 \mathrm{M}$ Tris, $\mathrm{pH}$ 7.5) applied on an uncoated glass substrate.

a)

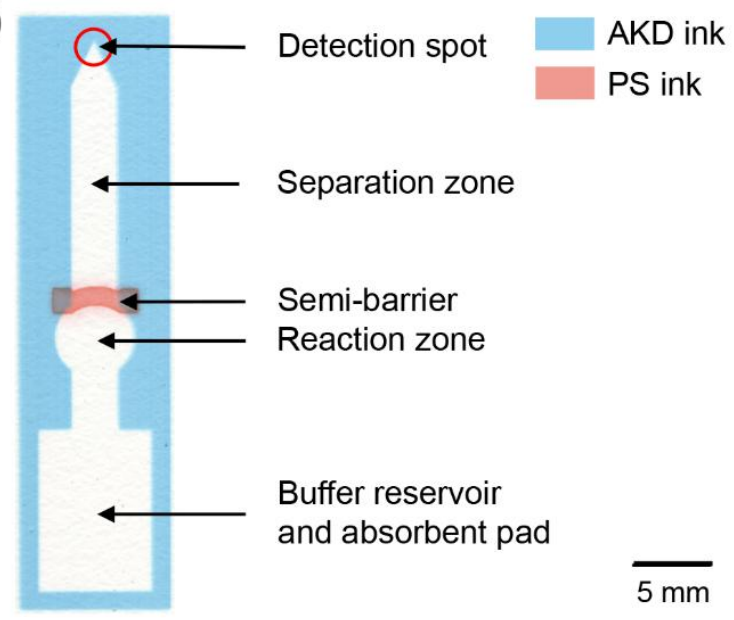

b)

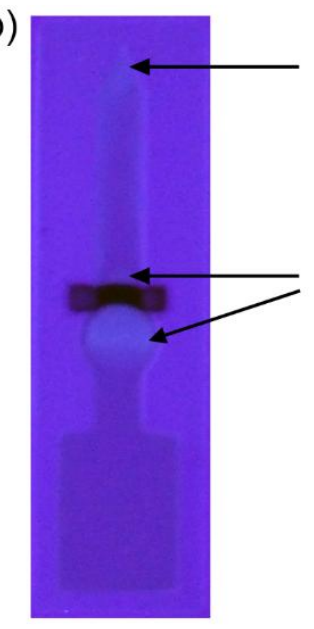

Metabolite elutes together with the solvent front

Co-substrate is retained by the FCC coating
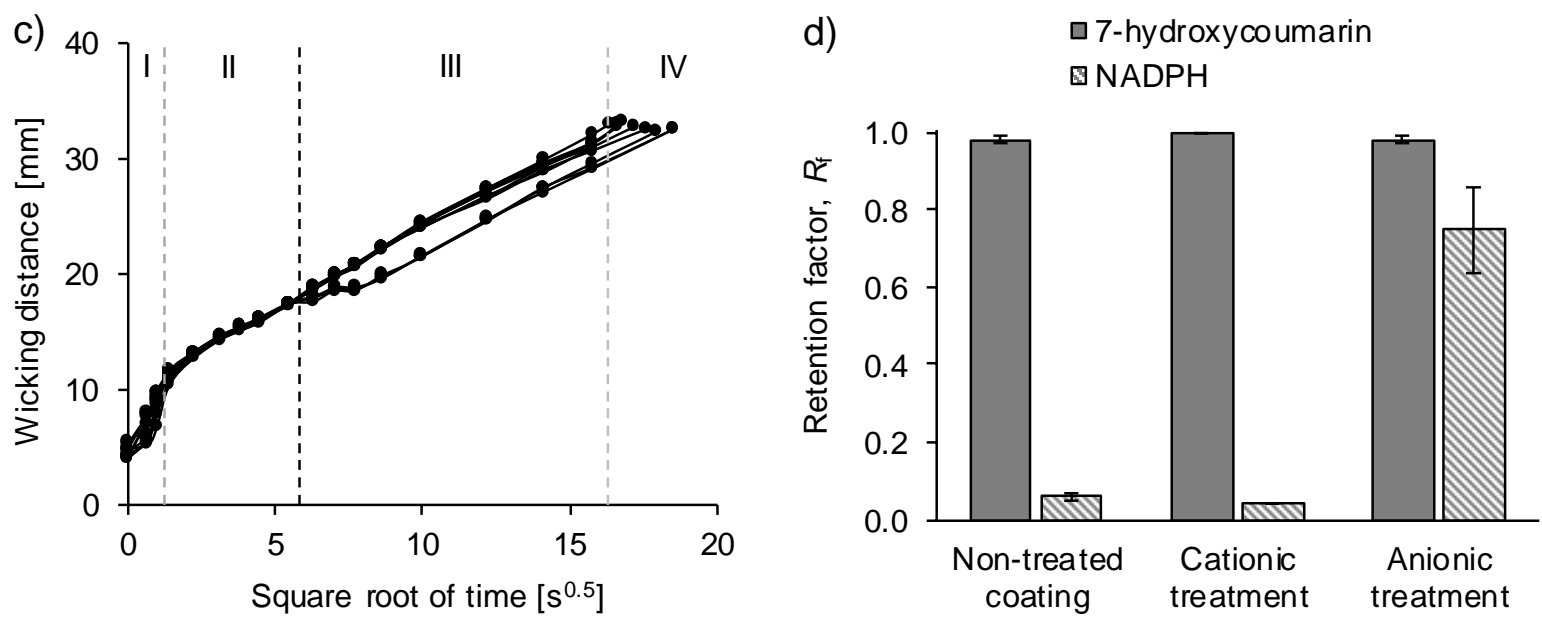

Figure 4. a) Photograph of the final assay design illustrating the different operation zones implemented on the FCC substrates by inkjet printing and detected area marked with a red ring. b) Photograph of the assay under UV lamp after an elution experiment illustrating the separation of the highly fluorescent cosubstrate (NADPH) and the faint fluorescent metabolite (7hydroxycoumarin). c) The capillary wicking distance as a function of square root of time on the final assay ( $n=10$ individual assays). The dash lines indicate the borders of the different zones on the final assay (semi-barrier marked with a black dash line): (I) liquid reservoir, (II) reaction zone, (III) separation zone, and (IV) detection spot. d) The retention factors $\left(R_{\mathrm{f}}\right)$ of 7 hydroxycoumarin and NADPH when applied on top of non-treated FCC containing 5 pph MFC $\mathrm{B}$ or on cationically (polyDADMAC) or anionically (NaPa) treated FCC coating $(n=3)$. 


\section{WILEY-VCH}
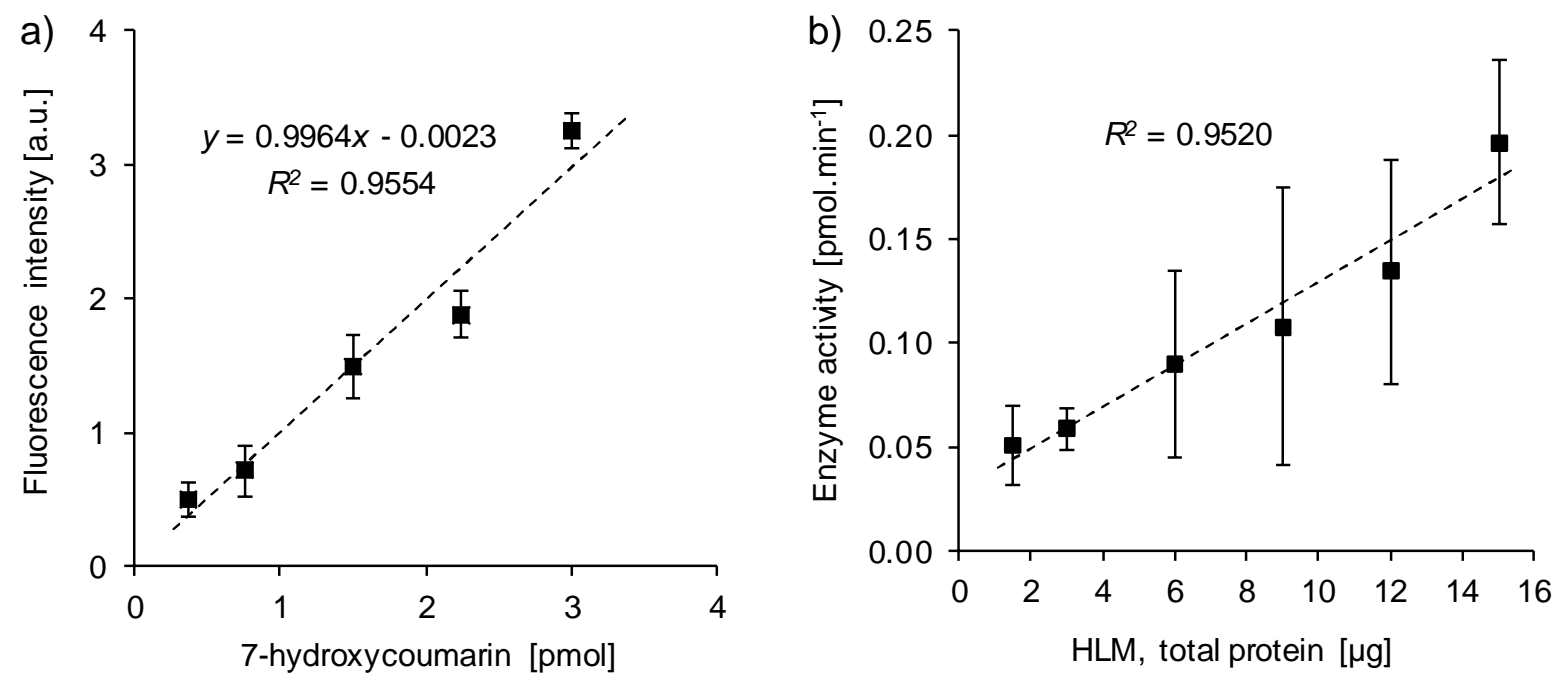

c)

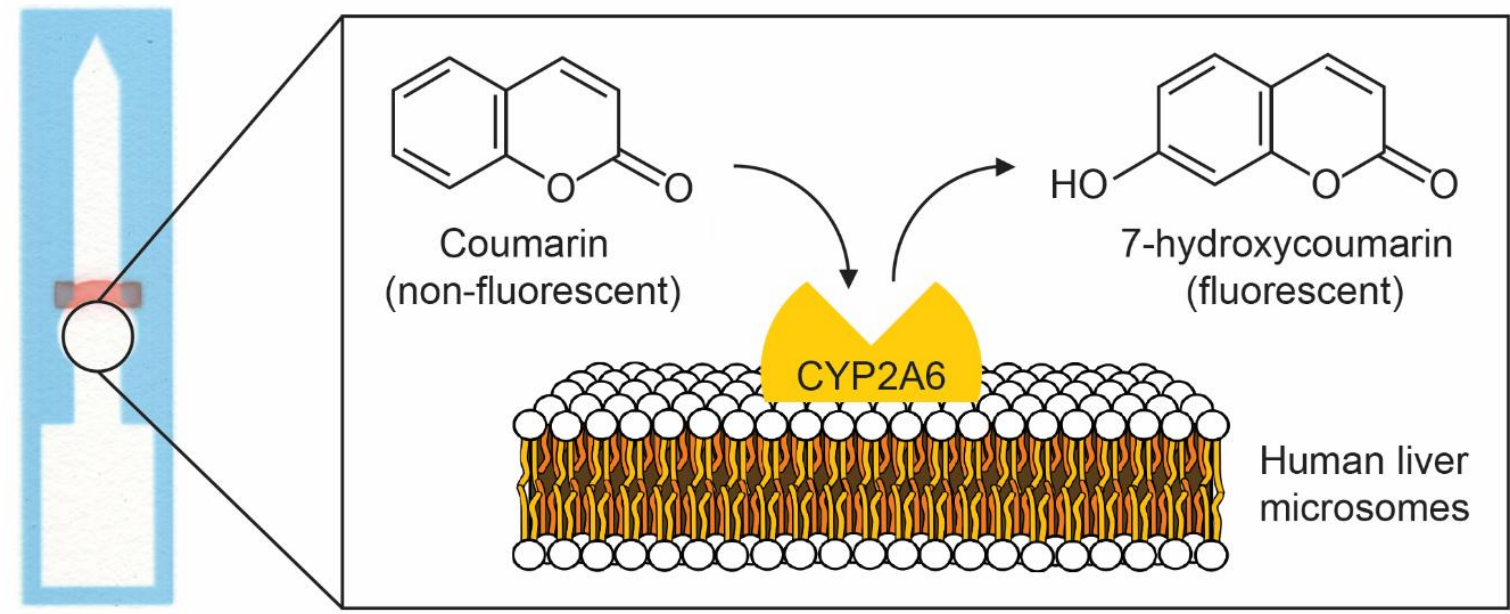

Figure 5. a) Calibration curve of the final assay, b) enzyme activity and c) a schematic of coumarin 7-hydroxylation taking place on a lateral flow assay. 


\section{WILEY-VCH}

Human phase I metabolism interactions mediated by cytochrome $\mathbf{P 4 5 0}$ can be studied using a microfluidic lateral flow assay constructed from functionalized calcium carbonate (FCC) and microfibrillated cellulose (MFC). The FCC-based platform coupled with fluorescence detection offers a viable method for studying CYP enzyme interactions in vitro, at comparatively low cost but with significantly increased selectivity compared to conventional well-plate assays.

Keywords: porous materials, functional coatings, inkjet printing, drug metabolism, cytochrome P450

Eveliina Jutila*, Risto Koivunen, Iiro Kiiski, Roger Bollström, Tiina Sikanen*, Patrick Gane

Microfluidic Lateral Flow Cytochrome P450 Assay on Printed Functionalized Calcium Carbonate-based Platform for Rapid Screening of Human Xenobiotic Metabolism

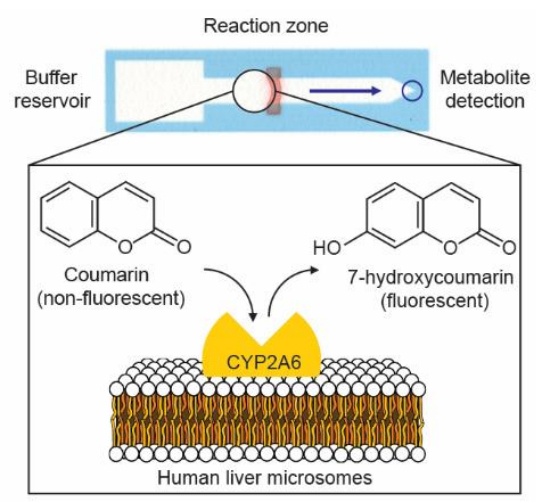

